\title{
Prevalence of drug resistant HIV-1 in rural areas of Hubei province in the People's Republic of China
}

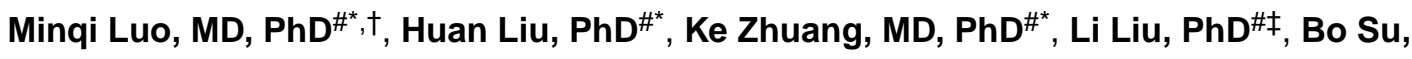 \\ $\mathrm{PhD}^{\star}$, Rongrong Yang, $\mathrm{MD}^{\dagger}$, Po Tien, $\mathrm{PhD}^{\star}$, Linqi Zhang, $\mathrm{PhD}^{\S}$, Xien Gui, $\mathrm{MD}^{\dagger}$, and Zhiwei \\ Chen, PhD, DVM ${ }^{\ddagger}$ \\ "Modern Virology Research Center and AIDS Center, State Key Laboratory of Virology, College of \\ Life Sciences, Wuhan University, Hubei 430072, P.R. China \\ †Zhongnan Hospital, Wuhan University, Wuhan 430071, China \\ $\ddagger$ AIDS Institute, The University of Hong Kong Li Ka Shing Faculty of Medicine, Hong Kong SAR, \\ P.R. China \\ §AIDS Research Center, Chinese Academy of Medical Sciences Peking Union Medical College, \\ Beijing 100730, P.R. China. \\ \# These authors contributed equally to this work.
}

\section{Abstract}

Objective-To determine the prevalence of drug resistant HIV-1 and the efficacy of first-line HAART regimens consisted of generic NRTI and NNRTI among 339 study subjects in rural areas of Hubei province, China.

\begin{abstract}
Methods-Two cross-sectional studies were conducted to investigate 150 HAART-naïve (99 received subsequent therapy) between 2003 and 2005 and 288 HAART-experienced patients mainly between 2005 and 2006. Patients' CD4 ${ }^{+} \mathrm{T}$ cell count and viral load were determined. HIV-1 pol gene fragments were amplified from patients' plasma by RT-PCR, subsequently sequenced and analyzed.
\end{abstract}

Results- $83.5 \%$ of the patients were from rural villages. They were dominantly infected with subtype B' HIV-1 (96.7\%) through paid blood donation (64.6\%) and related blood transfusion (28.3\%). We found that there was a steady increase of CD4 count over time among treated patients without detectable viral load $(186 / 288,64.6 \%)$. There was, however, an increasing prevalence of NRTI and NNRTI resistant mutations among patients with detected viremia $(102 / 288,35.4 \%)$ after treatment for 3-6 (24.3\%), 9-12 (57.1\%) and 20-24 (63.3\%) months, respectively. The increasing rates were associated with significant CD4 count drop and viral load increase. Some patients also developed multi-drug resistant mutants.

Conclusions-We report the first HIV-1 drug resistance study after two-years on HAART among Chinese patients living in rural villages. Our data suggest that a significant portion of

Correspondence to: Zhiwei Chen, Ph.D.,DVM, AIDS Institute, The University of Hong Kong Li Ka Shing Faculty of Medicine; 21 Sassoon Road, Pokfulam, Hong Kong SAR, P.R. China, Tel (852) 2819 9831; Fax (852) 2817 7805; zchenai@ @ku.hk.

Conflicts of interests: None. 
patients are failing first-line regimens with a trend of AIDS progression. It is therefore necessary to maximize the drug adherence and to make affordable second-line HAART regimens available immediately. Our results have implications for implementing HAART in under-resourced developing country settings.

\section{Keywords}

HIV-1; HAART; Drug Resistance; subtype B'; China

\section{Introduction}

Human immunodeficiency virus type 1 (HIV-1) infection has spread throughout China mainly through illegal intravenous drug use, illegal blood donation/plasma transfusions and sexual contact. ${ }^{1.5}$ Nowadays, there are an estimated 700,000 Chinese people living with HIV-1 including 85,000 acquired immunodeficiency syndrome (AIDS) patients. In 2007 alone, there were around 50,000 new HIV infections and 20,000 AIDS deaths in China. Importantly, more than half of these newly infected individuals acquired HIV-1 infections through sexual transmission (heterosexual 44.7\% and MSM 12.2\%), suggesting that the virus is rapidly spreading from high-risk groups into the general population in recent years (Ministry of Health China, 2007; http://www.moh.gov.cn/newshtml/20703.htm). Given China's 1.3 billion population, effective measures have to be implemented to reduce the ongoing transmission of HIV-1 infection.

While strengthening HIV-1 prevention efforts, China bears the load of an increasing number of symptomatic patients. We have previously reported the finding of a large number of subtype B' HIV-1 infections in Central China region including Henan and Hubei provinces. ${ }^{2}{ }^{4}$ Over half of these patients, who became infected through illegal blood donations in the 1990s, have become symptomatic and developed AIDS in the past years, creating an urgent need for highly active antiretroviral therapy (HAART) to reduce AIDSrelated mortality. Under these circumstances, the Chinese government launched the nationwide "Comprehensive AIDS Response (CARES) Program", also called the "Four Frees and One Care" program: free HAART for all rural AIDS patients and urban residents in financial difficulty, free voluntary counseling and testing (VCT), free education to children orphaned by AIDS, free prevention of mother-to-child transmission (PMTCT) services for pregnant women, and care to the households of people living with HIV/ AIDS. ${ }^{6}, 7$ Over thirty thousand AIDS patients have been treated with HAART under the program since its inception in 2003.

Despite the positive intentions of the government in providing free treatment to its citizens, treating thousands of AIDS patients without sufficient monitoring and management in rural villages has the international and domestic clinical community worried about the outcomes. With HAART, treatment failure and development of viral resistant mutants remain significant problems globally. ${ }^{8}$ In China, as in many other developing countries, there has been a lack of cost-effective second-line cost HAART regimens. ${ }^{6}, 9,10$ Moreover, the majority of infected people live in rural villages where well-trained infectious disease doctors are not easily accessible. These factors have posted great challenges to the nation. To 
date, there have been no published data on the prevalence of HIV-1 drug resistant strains in Hubei province, home to over 60 million people. By the end of 2006, approximately 1211 AIDS patients received HAART. In this study, we report findings on the prevalence of drug resistant HIV-1 strains in Hubei by characterizing specimens of 339 patients.

\section{Materials and methods}

\section{Study subjects and sample collection}

Our study subjects were mainly from the four counties and municipalities in Hubei province with the highest concentration of AIDS patients, including Xiangfan, Suizhou, Xishui and Wuhan, 595 specimens were collected between 2003 and 2006. Of note, samples of treatment-naïve patients were mainly collected between 2003 and 2005 whereas those of treated patients were mainly obtained between 2005 and 2006. The patients were treated with first-line HAART regimens consisted of two nucleoside reverse transcriptase inhibitors (NRTIs; AZT+DDI or D4T+3TC) and non-nucleoside reverse transcriptase inhibitors (NNRTI; NVP or EFV) provided by the government. AZT, D4T, DDI, and NVP are generic drugs made in China while 3TC and EFV are imported. The generic drugs were manufactured following the guideline and regulation by the State Food and Drug Administration of China. No protease inhibitors (PIs) were available to our study subjects. All drugs were administered with doses based on standardized guidelines and the administration of the drugs was supervised by the local physicians. After signing informed consent, all patients were interviewed face-to-face by trained and qualified physicians with a standardized questionnaire. The promotion of drug adherence also gains assistance through community measures. For example, patients are organized into groups by local doctors or village leaders to monitor one another to take medicine on time each day. Our study protocols were approved by local hospital ethics committees. A total of 339 AIDS patients were examined in this study including 150 HAART-naïve, which included 99 who subsequently received treatment, and 288 HAART-experienced patients (Table 1). 288 HAART-experienced patients were treated with the first line ART drugs with the following regimens: AZT, DDI, NVP ( $\mathrm{n}=108)$; D4T, 3TC, NVP ( $\mathrm{n}=56)$; D4T, DDI, NVP ( $\mathrm{n}=25)$; AZT, DDI, EFV, NVP ( $\mathrm{n}=24)$; AZT, 3TC, NVP ( $\mathrm{n}=12)$; AZT, DDI, EFV ( $\mathrm{n}=11)$; D4T, DDI, EFV, NVP ( $\mathrm{n}=11)$; AZT, D4T, DDI, EFV, NVP ( $\mathrm{n}=6)$; AZT, D4T, DDI, 3TC, EFV, NVP ( $\mathrm{n}=5)$; D4T, DDI, EFV ( $n=5)$; AZT, 3TC, EFV (n=4); AZT, D4T, 3TC, NVP (n=4); AZT, D4T, DDI, NVP ( $n=3)$; D4T, 3TC, EFV (n=3); D4T, DDI, 3TC, NVP (n=3); AZT, D4T, DDI, 3TC, EFV (n=2); AZT, DDI, 3TC, EFV (n=2); AZT, DDI, 3TC, EFV, NVP (n=2); AZT, D4T, 3TC, EFV $(n=1)$ and AZT, D4T, DDI, 3TC, NVP $(n=1)$. We tested all samples which were made available to us with patients' consent for resistance testing. By the end of 2006, the accumulated number of treated patients was 1211 in Hubei province. We therefore have studied 24\% (288/1211) of patients who received HAART.

\section{Viral load measurement}

All plasma samples were subjected to HIV-1 viral load measurement. Plasma HIV-1 RNA was quantified using a fluorescent quantification reverse transcription (RT)-PCR Assay kit (Shenzhen PG Biotech Co. Ltd, P.R. China) using the Bio-Rad icycler IQ real time PCR 
machine (Bio-Rad, USA). The detection limit of this assay is $500 \mathrm{RNA}$ copies $/ \mathrm{ml}$. All specimens were quantified according to the manufacturer's instructions.

\section{$\mathrm{CD4}^{+} \mathrm{T}$ cell count}

The absolute number and the percentage of circulating $\mathrm{CD} 4^{+} \mathrm{T}$ lymphocytes were analyzed by flow cytometry (Beckman Coulter EPICS XL, California, USA), using fluorescent labeled monoclonal antibodies (Beckman Coulter, California, USA) specific for $\mathrm{CD}^{+} \mathrm{T}$ lymphocytes.

\section{Nucleic acid extraction, amplification, and sequencing}

HIV RNA was extracted and purified from $200 \mu \mathrm{l}$ plasma with Roche High Pure Viral RNA isolation kit according to the manufacturer's protocol. For synthesis of cDNA, reverse transcript reaction was run with 20 $\mu \mathrm{l}$ RNA, 40pmol downstream PCR primer zcrtB4 (5'GCCTCTGTTAATTGTTTTACATCATTAGTGTG, M-MLV reverse transcriptase (Promega, USA), dNTPs (Fermentas, USA), and ribonuclease inhibitor (HuaMei Company, PR China) for 60 minutes at $42^{\circ} \mathrm{C}$ in a thermal cycler (MJ-PTC200). A nested PCR strategy was employed to amplify a 1283-bp pol gene fragment including the complete protease gene and the first 313 codons of the RT gene. The primer sequences for the first round PCR were: ZcprtF0 5'-TTTAGCCTCCCTCAAATCACTCTTT (sense) and ZcrtB4 5'-GCC TCTGT TAATTGTTTTACATCATTAGTGTG (antisense); and for the second pairs were: ZcprtF1 5'-CTTCCCTCAAATCACTCTTTGGC (sense) and ZcrtB3 5'GCTCTTGATAAATTTGGTATGTCCATTG (antisense). The amplification cycles were $95^{\circ} \mathrm{C}$ for $2 \mathrm{~min}$ followed by 35 cycles of $95^{\circ} \mathrm{C}$ for $15 \mathrm{~s}, 55^{\circ} \mathrm{C}$ for $45 \mathrm{~s}$, and $72^{\circ} \mathrm{C}$ for $1.5 \mathrm{~min}$, plus the last extension of $72^{\circ} \mathrm{C}$ for $10 \mathrm{~min}$. The primers were designed according to all HIV-1 subtypes circulated in China. Amplified PCR products were purified using the PCR gel-purification kit (HuaMei Company, PR China) and were subjected to DNA sequencing after cloning into a TA vector (Invitrogen, USA) using an automated ABI 377 DNA sequencer (Applied Biosystems Inc., USA). The study was conducted in an HIV/AIDS research laboratory at Wuhan University under an internal quality control program. Multiple clones or samples were studied for individuals who harbor drug resistant genes.

\section{Analysis of HIV-1 drug-resistant mutations}

To eliminate potential PCR contamination, the sequences obtained were first compared to all known sequences in the HIV database by a BLAST search prior to analysis (http://hivweb.lanl.gov/content/index). ${ }^{11}$ Subsequently, the drug resistance analysis was conducted using tools in a public database (http://hivdb.stanford.edu). By comparing with the sequences in the database, the software enables the identification of the drug resistant mutations using a scoring system. ${ }^{12}$ Major drug-resistance mutations were defined as those which meet both the criteria of the International AIDS Society (IAS)-USA ${ }^{13}, 14$ and Stanford HIV Drug Resistance Database. ${ }^{15}, 16$

\section{Phylogenetic analysis of HIV-1 sequences}

To determine viral subtypes, HIV pol gene sequences were subjected to phylogenetic analysis using the neighbor-joining method of Kimura, implemented in the ClustalX1.81 
program as we previously described. ${ }^{1}, 4$ The genetic distance of the HIV-1 sequences was calculated using the two-parameter model of Kimura. The GenBank accession numbers of our sequences are pending.

\section{Statistical analysis}

The proportion of specimen anchoring drug-resistance mutations was expressed as a percentage of the total sample population examined. To test for statistical significance, we used the Wilcoxon two-sample rank sum test for continuous variables and the MantelHaenszel chi-square test for categorical variables. Fisher's exact test was also applied due to the small sample size. We used the computer program SPSS 11.0 for our statistical analyses.

\section{Results}

\section{Characteristics of study subjects}

A total of 339 AIDS patients were examined in this study including 150 HAART-naïve, which included 99 who subsequently received treatment, and 288 HAART-experienced patients (Table 1). For the latter, 186 had undetectable viral load over the course of treatment whereas 102 had detectable viremia. Our study subjects were mainly from four distinct geographic locations including Wuhan, Suizhou, Xishui and Xiangfan (Table 1 and Figure 1), where most of infection were identified in Hubei province (Table 1). ${ }^{4}$ One critical characteristic of our study subjects is that most of them $(283 / 339,83.5 \%)$ were found living in rural villages where the number of physicians specialized in infectious diseases is small. To promote the drug adherence, the daily outpatient treatment of AIDS patients also gained assistance through village or community measures such as organizing patients into groups to monitor one another to take medicine on time.

Consistent to our previous findings, paid blood donation $(64.6 \%, 219 / 339)$ or related blood transfusion $(28.3 \%, 96 / 339)$ were the major risk factors. A small percentage of the subjects acquired infection via sexual transmission $(7.1 \%, 24 / 339)$. By analyzing viral pol sequences of 152 study subjects with detectable viral load, we found that $96.7 \%$ (147/152) of them were infected with subtype B' HIV-1 strains (Fig.1), again consistent with our previous reports. The rest were infected with other viral subtypes (01_AE, $\left.n=4 ; 08 \_B C, n=1\right)$ (Fig. 1). Interestingly, all four 01_AE infected subjects acquired HIV-1 through sexual contacts.

\section{Treatment efficacy}

The viral load and CD4 counts were measured at the time of sample collection. Since we found that 186 patients had undetectable viral load during HAART for two years, the overall rate of viral suppression among treated patients was $64.6 \%$ (186/288). The remaining $35.4 \%$ $(102 / 288)$ of treated patients had detectable viral load at least once and therefore become the major target population for the subsequent drug resistance studies. On average, there was a significant increase of $\mathrm{CD} 4^{+} \mathrm{T}$ cell count in the HAART-experienced patients without detectable viral load $(231.8 \pm 128.6)$ when compared with the HAART-naïve patients (107.9 \pm 94.7$)$ (Table 1, $t$ Test, $p<0.05$ ). HAART among these patients therefore was rather effective. 
For HAART-experienced patients with detected viral load, the situation was complex. To further understand treatment efficacy, treated patients with and without detectable viral load were analyzed based on four groups: before HAART as well as 3-6, 9-12, and 20-24 months after HAART. For treated patients without detectable viral load, there was a clearly higher level of $\mathrm{CD}^{+}{ }^{+} \mathrm{T}$ cells (Fig. 2A). In contrast, among treated patients with detected viral load, there was a clear lower CD4 count and increasing viral load especially by 20-24 months post HAART initiation (Fig. 2A and 2B). There was a significant drop of CD4 count when compared with treated patients without detectable viral load at this time ( $t$ Test, $p<0.01$ ) (Fig. 2A). The average CD4 count among these patients dropped close to the level of untreated AIDS patients.

\section{HIV-1 drug resistance mutations}

We were able to amplify pol gene fragments from plasma samples collected from 123 of the 150 drug naïve patients (Table 1$)$. We found that ten subjects $(8.1 \%, 10 / 123)$ had drug resistant mutations including NRTI mutations $\mathrm{K} 65 \mathrm{R}(\mathrm{n}=2)$ and M184V $(\mathrm{n}=2)$ as well as NNRTI mutations K103N ( $\mathrm{n}=2)$, V106A $(\mathrm{n}=1), \mathrm{Y} 181 \mathrm{C}(\mathrm{n}=2)$ and Y188C $(\mathrm{n}=1)($ Table 2). The clonal frequency of mutations in each patient was K103N (1/2), V106A (1/5), Y181C (1/5)+M46I (1/5), Y188C (1/3), Y181C (1/5), K65R (1/3), M184V (1/4), M184V (1/3), K65R (1/5) and K103N (3/3), respectively. Nine patients had only one of these mutations whereas one individual had both M46I and Y181C. M46I is an PI mutation. Both patients with the K65R mutation were infected with subtype B' HIV-1. To understand whether these mutations were related to sequence polymorphism, we sequenced multiple clones of each subject. We found that only one patient had dominant K103N (100\%) whereas the rest of the mutations were less than $30 \%$ in each patient. Of note, five of the ten patients were not enrolled into our subsequent treatment programs.

The overall prevalence of drug-resistant variants increased significantly among patients with detected viremia after HAART for 3-6 (24.3\%), 9-12 (57.1\%) and 20-24 (63.3\%), respectively (Table 2). While there was an increasing frequency of NRTI resistant mutations over time associated with duration on HAART (10.8\% to $14.3 \%$ to $33.3 \%)$, the frequency of NNRTI resistant mutations increased at a higher rate (16.2\% to $51.4 \%$ to $60 \%)$. The mutations identified were dominating (100\%) the viral quasispecis in each individual by sequencing multiple clones. Clearly, the longer duration on HAART, the higher was the frequency of drug resistant mutations detected among these study projects. Importantly, the frequency of multi-drug resistance (MDR) to both NRTI and NNRTI had the similar trend of increasing over time (2.7\% to $8.6 \%$ to $23.3 \%)$. Since no PIs were used in first-line regimens, we did not detect any primary resistance mutations to PIs. Of five patients infected with nonsubtype B virus, one 01_AE patient developed M184V mutation and the 08_BC subject had V75A mutation, both after 6 months of HAART.

The spectrum of drug resistant mutations to NRTIs became broader overtime (Table 3 ). The initial NRTI mutations were confined to K65R and M184V/I that did not seem to increase significantly in frequency overtime. However, more mutations, including M41L, F116Y, Q151M, L210W and T215Y/F, were identified by 9-12 months. Subsequently, additional mutations D67N, K70R, L74V, V75M, F77L, Y115F and K219E were found by 20-24 
months. Since most of these NRTI mutations (12/14) were not found among HAART-naïve patients, we suggest that they emerged due to HAART. As for mutations to NNRTI, although the spectrum of drug resistance variants did not seem to change much overtime, the frequency of each mutation increased significantly (Table 3). The increase took place especially after treatment for 9-12 months for mutations K103N (28.6\%), V106A (2.9\%), Y181C (8.6\%), Y188C/L (8.6\%) and G190A/S (14.3\%) (Table 3). Since four of these five mutations (K103N, Y181C, Y188C/L and G190A/S) confer high level resistance to the first generation of NNRTIs, these mutations could play a critical role for the failure of HAART. Lastly, the frequency of resistance mutations to individual drugs was also investigated. We found that the frequency of resistance mutations increased significantly against AZT (23.3\%), D4T (23.3\%), DDI (20\%), NVP (60\%) and EFV (50\%) but seemingly not strongly against 3TC $(6.7 \%)$ after two years of treatment. We also found that many mutations might confer cross-resistance to other drugs, which were not used in the study area, potentially increasing the frequency of resistance mutations to NRTIs (e.g. ABC, TDF and FTC) and NNRTIs (e.g. DLV) simultaneously.

\section{First-line HAART Regimens}

288 HAART-experienced patients were treated with various first-line regimens as described in the "Materials and Methods" section. To understand which regimen is more effective in terms of viral suppression among Chinese patients, we further investigated the proportion of treated patients who received different HAART regimens and developed HIV resistance mutations. We analyzed every first-line regimen, which was used for at least ten subjects for up to two years. The prevalence of HIV resistance mutations for each regimen are as follows: AZT, DDI, NVP (32.4\%, $\mathrm{n}=35 / 108)$; D4T, 3TC, NVP (5.4\%, $\mathrm{n}=3 / 56)$; D4T, DDI, NVP (40\%, n=10/25); AZT, DDI, EFV, NVP (33\%, n=8/24); AZT, 3TC, NVP (16.7\%, $\mathrm{n}=2 / 12)$; AZT, DDI, EFV (45.4\%, n=5/11); D4T, DDI, EFV, NVP (45.4\%, n=5/11). As mentioned above, resistance mutations to $3 \mathrm{TC}$ are relatively rare among the study subjects investigated. Moreover, regimen D4T, 3TC and NVP seems more effective $(p<0.05)$ than other regimens in terms of viral suppression among Chinese patients treated for two years.

\section{DISCUSSION}

As HAART for HIV-1 infection becomes increasingly available in resource-limited settings, concerns regarding the emergence and spread of drug resistant mutants are mounting. ${ }^{17}, 18$ In this paper, we report a large scale study of HIV-1 drug resistance in a resource-limited setting in China because the majority of our study subjects $(283 / 339,83.5 \%)$ were from rural villages. ${ }^{4}$ In this setting, the prevalence of HIV-1 drug resistant variants among Chinese patients after two years of first-line HAART regimens remains unknown. Also unclear was how quickly Chinese patients would progress to AIDS if they failed these regimens. The present study was based on the characterization of specimens collected from 339 study subjects between 2003 and 2007. We found that the majority of study subjects were infected with subtype B' HIV-1 through paid blood donation or related blood transfusion, which was consistent to our previous report on AIDS epidemic in Central China region. ${ }^{2-4}$ The combination of B' viral subtype and Chinese host's genetic background has likely provided a 
unique situation for the understanding of HIV-1 drug resistance in a particular geographic location in the world.

The prevalence of HIV-1 drug resistance variants was low among our study subjects before the initiation of HAART. Genotype-resistance testing of chronically HIV-infected, antiretroviral-naive patients at the time of diagnosis has been suggested to become the standard of care. ${ }^{19}$ By analyzing HIV pol sequences of 123 HAART-naïve patients, we found ten subjects harboring drug-resistant variants $(8.1 \%, 10 / 123)$. This rate is slightly lower than that (13.9\%) recently found in neighboring Henan province. ${ }^{20}$ Moreover, these variants appeared to be a minor population of viral quasispecies at the time of testing because the frequency of resistance mutations was less than $30 \%$ among nine patients except for one who had $100 \% \mathrm{~K} 103 \mathrm{~N}$ mutation. This is in contrast to HAART-experienced patients, whose resistance mutations often dominated the viral quasispecies $(100 \%)$. The frequency of MDR variants was low as only one subject had displayed a low frequency of both M46I and Y181C. Based on these findings, we concluded that the majority of our study subjects $(113 / 123,91.9 \%)$ were truly HAART-naïve. Given that no HAART was provided to any of these study subjects, the finding of ten subjects with minor proportion of resistance mutants is probably due to the sequence polymorphism during the natural course of HIV-1 evolution. We however cannot absolutely exclude the possibility that some mutations may arise from PCR amplification despite high fidelity enzyme and proper quality controls were used in the experiments. It is possible that some patients could have obtained some anti-HIV drug through private source and did not inform us. This situation may be applied to four subjects, who had mutations of V106A $(n=1), Y 181 C(n=2)$ and Y188C $(n=1)$, respectively, because these mutations have not been documented among untreated patients infected with subtype B HIV-1 anywhere in the world. ${ }^{16}$ It is also possible (though perhaps unlikely) that these patients were infected with HIV resistant strains acquired from another individual previously exposed to treatment.

The increasing prevalence of HIV-1 drug resistance variants was responsible for the failure of HAART among 35.5\% (102/288) of our study subjects. After receiving continuous HAART for at least three months, we found a steady increase of CD4 count over time among treated patients without detectable viral load (185/288, 64.5\%). There were, however, 102 patients who had detectable viremia with a mean viral load close to that of the untreated persons ( $\log 10,4.75 \pm 0.94$ vs $5.44 \pm 1.04$; Table 1 ). This finding prompted us to conduct further studies to investigate the underlying mechanism in relation to drug resistance mutations. We found that there was an increasing prevalence of dominant NRTI and NNRTI resistance mutations among patients with detected viremia $(102 / 288,35.5 \%)$ after treatment for 3-6 (24.3\%), 9-12 (57.1\%) and 20-24 (63.3\%) months, respectively (Table 2). In particular, the prevalence of mutations, which confer high level resistance to NNRTIs, increased over 50\% after 9-12 months of HAART, which was consistent to previous findings in the neighboring Henan province. ${ }^{20}, 21,22$ It is possible that the extensive use of regimen AZT+DDI+NVP could have resulted in this outcome. ${ }^{20}, 22,23$ Furthermore, the spectrum of drug resistant mutations to NRTIs also became broader over time (Table 3), and most of these NRTI mutations (12/14) were not found among HAART-naïve patients. Taken together, our findings suggested that the emergence of drug resistance mutations must have played a role in the failure of HAART observed. 
The selection of a fully suppressive regimen is critical to reduce the prevalence of HIV resistance mutations. HAART exerts selective pressure for HIV resistance if the existing regimen is not fully suppressive. Due to the increasing demand of HAART in China, the selection of a cost-effective regimen has been a serious issue for fulfilling the "Four Frees and One Care" commitment in China. Since the generic AZT+DDI+NVP regimen was readily available, it was practical to use the AZT+DDI+NVP regimen as the first choice. However, with the rapidly increasing number of resistance mutations to this regimen over time, it is necessary to consider other regimens. ${ }^{24}$ Here, we found that resistance mutations to 3TC (e.g. M184V/I, K65R) are relatively rare (Table 3) and the D4T+3TC+NVP regimen seems more effective than other regimens in terms of minimizing drug resistance among Chinese patients on HAART for two years (see results). If the results were not biased by the drug adherence rate and optimal dosing of each regimen ${ }^{25}$, whether or not the use of D4T $+3 \mathrm{TC}+\mathrm{NVP}$ should be considered as the major first-line regimen for HAART naïve Chinese patients will require further careful investigation giving its higher cost, manageable toxicity issue and unsuccessful experience in other nations. ${ }^{26} 28$ Up to now, we have not found primary PI mutations among patients failing HAART because most of them were still PInaïve (Table 3). Second-line regimens therefore should include PIs, which must be made available or affordable to patients under a resource limited setting ${ }^{17}$. The implementation of second-line regimens will be important to minimize the transmission of drug-resistant viruses especially those with MDR in China. ${ }^{29} 31$ With the evolving HIV-1 epidemic in China, it becomes necessary to monitor HIV-1 resistant variants to antiretroviral agents in newly infected individuals. ${ }^{27}, 32,33$

Progression to AIDS may take place within two years under HAART if Chinese patients no longer respond to first line HAART regimens. One of the most critical findings of this study was the significant drop of CD4 count by 20-24 months post HAART among treated patients with increasing viral load, when compared with treated patients without detectable viral load (Fig. 2A and 2B). Since the average number of CD4 count has dropped to the level which is similar to that of untreated AIDS patients, our data have provided direct evidence that a significant portion of treated patients were failing on HAART and have clinically progressed toward AIDS. There is therefore an urgent need for second-line HAART regimens for these patients. While life-saving second-line medication (like ritonavir-boosted lopinavir and tenofovir) are now available on the market, ${ }^{34}, 35$ their prices put them out of reach for most Chinese AIDS patients and the government of China has not yet included them in the free treatment program. To this end, the preservation of first-line regimens through maximizing the drug adherence must become the top priority work of the ongoing HAART. ${ }^{36}, 37$ Our results have provided critical information on HIV-1 drug resistance variants among Chinese patients living in rural villages after two-year on HAART, and may have implications for implementing HAART in developing countries with limited resources. ${ }^{17}, 25,26$

\section{ACKNOWLEDGEMENTS}

This study was supported by China AIDS Initiative and the National Basic Research Program of China (973 project 2006CB504208). We acknowledge the University Development Fund of the University of Hong Kong to its AIDS Institute as well as the NIH R01 grant HL080211-01 (to ZC). We also thank David D. Ho for scientific advice and Kathrine Meyers for editorial inputs and discussions. 


\section{REFERENCES}

1. Zhang Y, Lu L, Ba L, et al. Dominance of HIV-1 subtype CRF01_AE in sexually acquired cases leads to a new epidemic in Yunnan province of China. PLoS Med. Nov.2006 3(11):e443. [PubMed: 17105339]

2. Zhang L, Chen Z, Cao Y, et al. Molecular characterization of human immunodeficiency virus type 1 and hepatitis $\mathrm{C}$ virus in paid blood donors and injection drug users in china. J Virol. Dec; 2004 78(24):13591-13599. [PubMed: 15564470]

3. Zhuang K, Gui X, Su B, Tien P, Chen Z, Zhang L. High prevalence of HIV infection among women and their children in Henan Province, China. J Acquir Immune Defic Syndr. Aug 15; 2003 33(5): 649-650. [PubMed: 12902811]

4. Su B, Liu L, Wang F, et al. HIV-1 subtype B' dictates the AIDS epidemic among paid blood donors in the Henan and Hubei provinces of China. Aids. Nov 21; 2003 17(17):2515-2520. [PubMed: 14600524]

5. Zhao R, Gao H, Shi X, et al. Sexually transmitted disease/HIV and heterosexual risk among miners in townships of Yunnan Province, China. AIDS Patient Care STDS. Dec; 2005 19(12):848-852. [PubMed: 16375617]

6. Cao YZ, Lu HZ. Care of HIV-infected patients in China. Cell Res. Nov-Dec;2005 15(11-12):883890. [PubMed: 16354564]

7. Zhang F, Au MC, Bouey PD, et al. The diagnosis and treatment of HIV-infected children in China: challenges and opportunities. J Acquir Immune Defic Syndr. Apr 1; 2007 44(4):429-434. [PubMed: 17224849]

8. Lindstrom A, Ohlis A, Huigen M, et al. HIV-1 transmission cluster with M41L 'singleton' mutation and decreased transmission of resistance in newly diagnosed Swedish homosexual men. Antivir Ther. 2006; 11(8):1031-1039. [PubMed: 17302373]

9. Zhang FJ, Maria A, Haberer J, Zhao Y. Overview of HIV drug resistance and its implications for China. Chin Med J (Engl). Dec 5; 2006 119(23):1999-2004. [PubMed: 17199945]

10. Zhang FJ, Pan J, Yu L, Wen Y, Zhao Y. Current progress of China's free ART program. Cell Res. Nov-Dec;2005 15(11-12):877-882. [PubMed: 16354563]

11. Kuiken C, Korber B, Shafer RW. HIV sequence databases. AIDS Rev. Jan-Mar;2003 5(1):52-61. [PubMed: 12875108]

12. Rhee SY, Gonzales MJ, Kantor R, Betts BJ, Ravela J, Shafer RW. Human immunodeficiency virus reverse transcriptase and protease sequence database. Nucleic Acids Res. Jan 1; 2003 31(1):298303. [PubMed: 12520007]

13. Johnson VA, Brun-Vezinet F, Clotet B, et al. Update of the drug resistance mutations in HIV-1: 2007. Top HIV Med. Aug-Sep;2007 15(4):119-125. [PubMed: 17720996]

14. Johnson VA, Brun-Vezinet F, Clotet B, et al. Update of the drug resistance mutations in HIV-1: Fall 2006. Aug-Sep;Top HIV Med. 2006 14(3):125-130.

15. Shafer RW. Rationale and uses of a public HIV drug-resistance database. J Infect Dis. Sep 15; 2006 194(Suppl 1):S51-58. [PubMed: 16921473]

16. Shafer RW, Rhee SY, Pillay D, et al. HIV-1 protease and reverse transcriptase mutations for drug resistance surveillance. Aids. Jan 11; 2007 21(2):215-223. [PubMed: 17197813]

17. Walensky RP, Weinstein MC, Yazdanpanah Y, et al. HIV drug resistance surveillance for prioritizing treatment in resource-limited settings. Aids. May 11; 2007 21(8):973-982. [PubMed: 17457091]

18. Spacek LA, Shihab HM, Kamya MR, et al. Response to antiretroviral therapy in HIV-infected patients attending a public, urban clinic in Kampala, Uganda. Clin Infect Dis. Jan 15; 2006 42(2): 252-259. [PubMed: 16355337]

19. Sax PE, Islam R, Walensky RP, et al. Should resistance testing be performed for treatment-naive HIV-infected patients? A cost-effectiveness analysis. Clin Infect Dis. Nov 1; 2005 41(9):13161323. [PubMed: 16206108]

20. Li JY, Li HP, Li L, et al. Prevalence and evolution of drug resistance HIV-1 variants in Henan, China. Cell Res. Nov-Dec;2005 15(11-12):843-849. [PubMed: 16354557] 
21. Li J, Lu JF, Dong HH, et al. Phenotypic resistance of resistant strains of HIV type-1 subtype B in China. Chin Med J (Engl). Dec 5; 2006 119(23):1972-1977. [PubMed: 17199941]

22. Ma L, Sun J, Xing H, et al. Genotype and phenotype patterns of drug-resistant HIV-1 subtype B' (Thai B) isolated from patients failing antiretroviral therapy in China. J Acquir Immune Defic Syndr. Jan 1; 2007 44(1):14-19. [PubMed: 17019361]

23. Conway B, Wainberg MA, Hall D, et al. Development of drug resistance in patients receiving combinations of zidovudine, didanosine and nevirapine. Aids. Jul 6; 2001 15(10):1269-1274. [PubMed: 11426071]

24. Clotet B. Strategies for overcoming resistance in HIV-1 infected patients receiving HAART. AIDS Rev. Jul-Sep;2004 6(3):123-130. [PubMed: 15595429]

25. Cingolani A, Antinori A, Rizzo MG, et al. Usefulness of monitoring HIV drug resistance and adherence in individuals failing highly active antiretroviral therapy: a randomized study (ARGENTA). Aids. Feb 15; 2002 16(3):369-379. [PubMed: 11834948]

26. Kamya MR, Mayanja-Kizza H, Kambugu A, et al. Predictors of long-term viral failure among ugandan children and adults treated with antiretroviral therapy. J Acquir Immune Defic Syndr. Oct 1; 2007 46(2):187-193. [PubMed: 17693883]

27. Tam LW, Hogg RS, Yip B, Montaner JS, Harrigan PR, Brumme CJ. Performance of a World Health Organization first-line regimen (stavudine/lamivudine/nevirapine) in antiretroviral-naive individuals in a Western setting. HIV Med. Jul; 2007 8(5):267-270. [PubMed: 17561871]

28. Zhou HY, Zheng YH, Zhang CY, et al. Evaluation of human immunodeficiency virus type 1infected Chinese patients treated with highly active antiretroviral therapy for two years. Viral Immunol. 2007; 20(1):180-187. Spring. [PubMed: 17425432]

29. Delaugerre C, Morand-Joubert L, Chaix ML, et al. Persistence of multidrug-resistant HIV-1 without antiretroviral treatment 2 years after sexual transmission. Antivir Ther. Jun; 2004 9(3): 415-421. [PubMed: 15259904]

30. Havlir D, Vella S, Hammer S. The Global HIV Drug Resistance Surveillance Program: a partnership between WHO and IAS. International AIDS Society. Aids. Jul 5; 2002 16(10):7-9. [PubMed: 12146462]

31. Shet A, Markowitz M. Transmitted multidrug resistant HIV-1: new and investigational therapeutic approaches. Curr Opin Investig Drugs. Aug; 2006 7(8):709-720.

32. Simon V, Vanderhoeven J, Hurley A, et al. Evolving patterns of HIV-1 resistance to antiretroviral agents in newly infected individuals. Aids. Jul 26; 2002 16(11):1511-1519. [PubMed: 12131189]

33. Markowitz M, Mohri H, Mehandru S, et al. Infection with multidrug resistant, dual-tropic HIV-1 and rapid progression to AIDS: a case report. Lancet. Mar 19-25; 2005 365(9464):1031-1038. [PubMed: 15781098]

34. Antinori A, Trotta MP, Nasta P, et al. Antiviral efficacy and genotypic resistance patterns of combination therapy with stavudine/tenofovir in highly active antiretroviral therapy experienced patients. Antivir Ther. 2006; 11(2):233-243. [PubMed: 16640104]

35. Dunn LA, Andrews KT, McCarthy JS, et al. The activity of protease inhibitors against Giardia duodenalis and metronidazole-resistant Trichomonas vaginalis. Int J Antimicrob Agents. Jan; 2007 29(1):98-102. [PubMed: 17137752]

36. Oyugi JH, Byakika-Tusiime J, Ragland K, et al. Treatment interruptions predict resistance in HIVpositive individuals purchasing fixed-dose combination antiretroviral therapy in Kampala, Uganda. Aids. May 11; 2007 21(8):965-971. [PubMed: 17457090]

37. Wanchu A, Kaur R, Bambery P, Singh S. Adherence to generic reverse transcriptase inhibitorbased antiretroviral medication at a Tertiary Center in North India. AIDS Behav. Jan; 2007 11(1): 99-102. [PubMed: 16607479] 


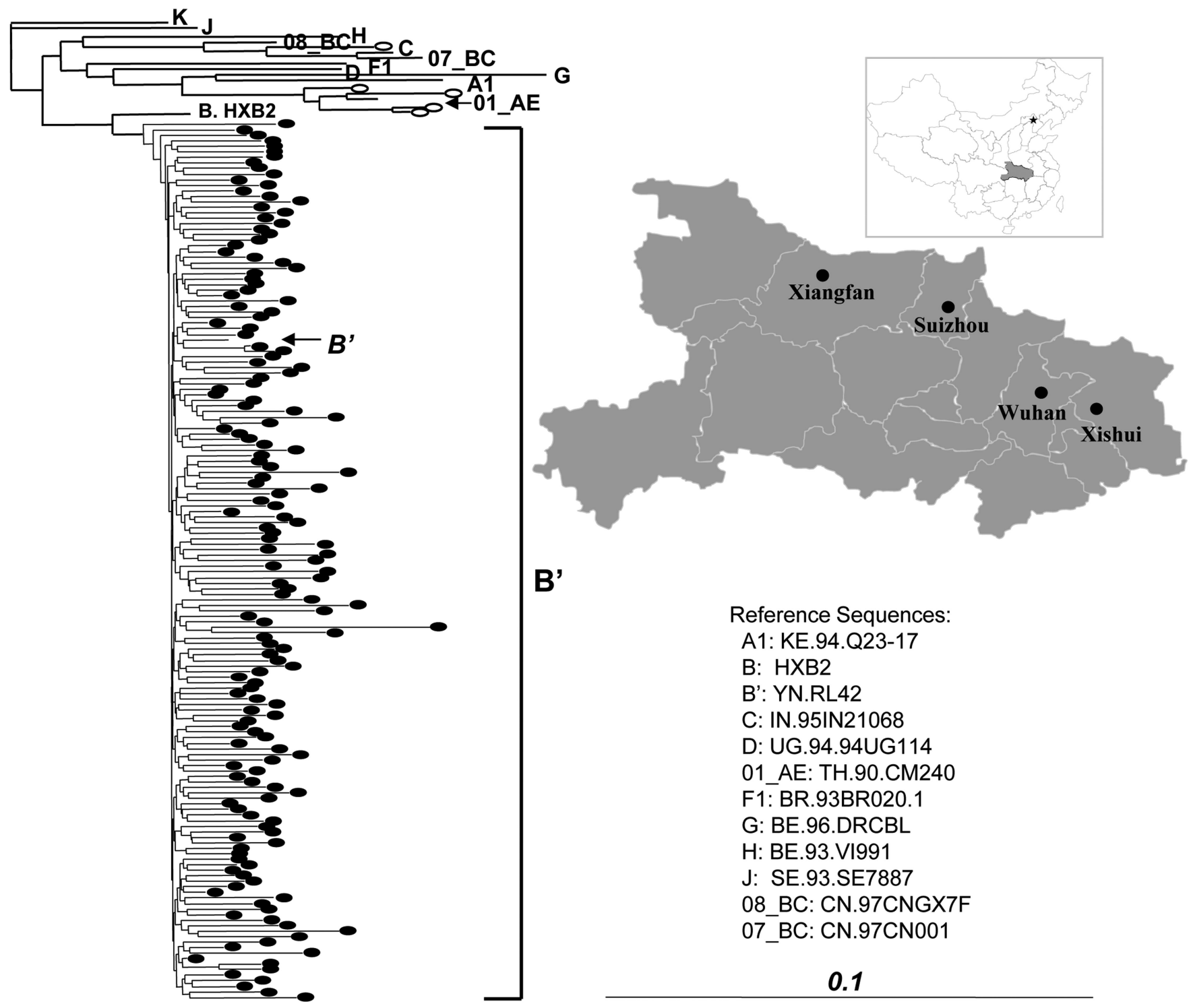

FIGURE 1.

Phylogenetic neighbor-joining tree for HIV pol sequences and map of Hubei province. The reference sequences for phylogenetic analysis are obtained from HIV Sequence Database. The horizontal branch is drawn in accordance with their relative genetic distances. The vertical lines are for clarity of the tree presentation. The oval symbols represent HIV-1 strains derived from our study subjects. Four study areas are shown in the Map of Hubei province. The inset is the map of China. 


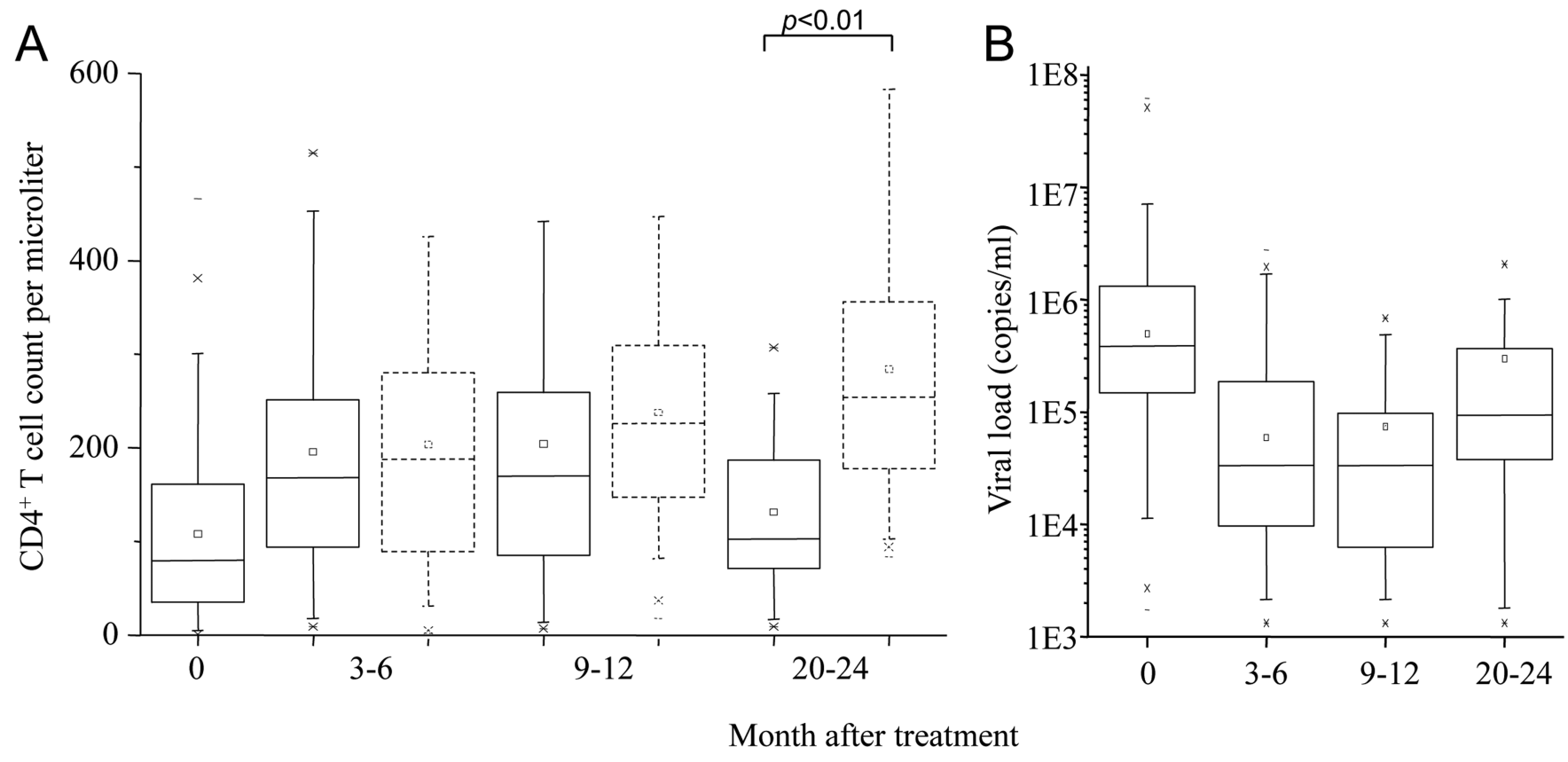

FIGURE 2.

Measurement of CD4 cell count (A) and HIV-1 viral load (B) of study subjects. Solid lines are for patients with detectable viral load. Dashes lines are for patients without detectable viral load $(<500$ copies $/ \mathrm{ml})$. The top and bottom of each rectangular box denote the $75^{\text {th }}$ and $25^{\text {th }}$ percentiles, respectively, with the median shown inside the box. Horizontal bars extending from each box represent the $99^{\text {th }}$ and $1^{\text {st }}$ percentiles. 


\section{롤 \\ 골}

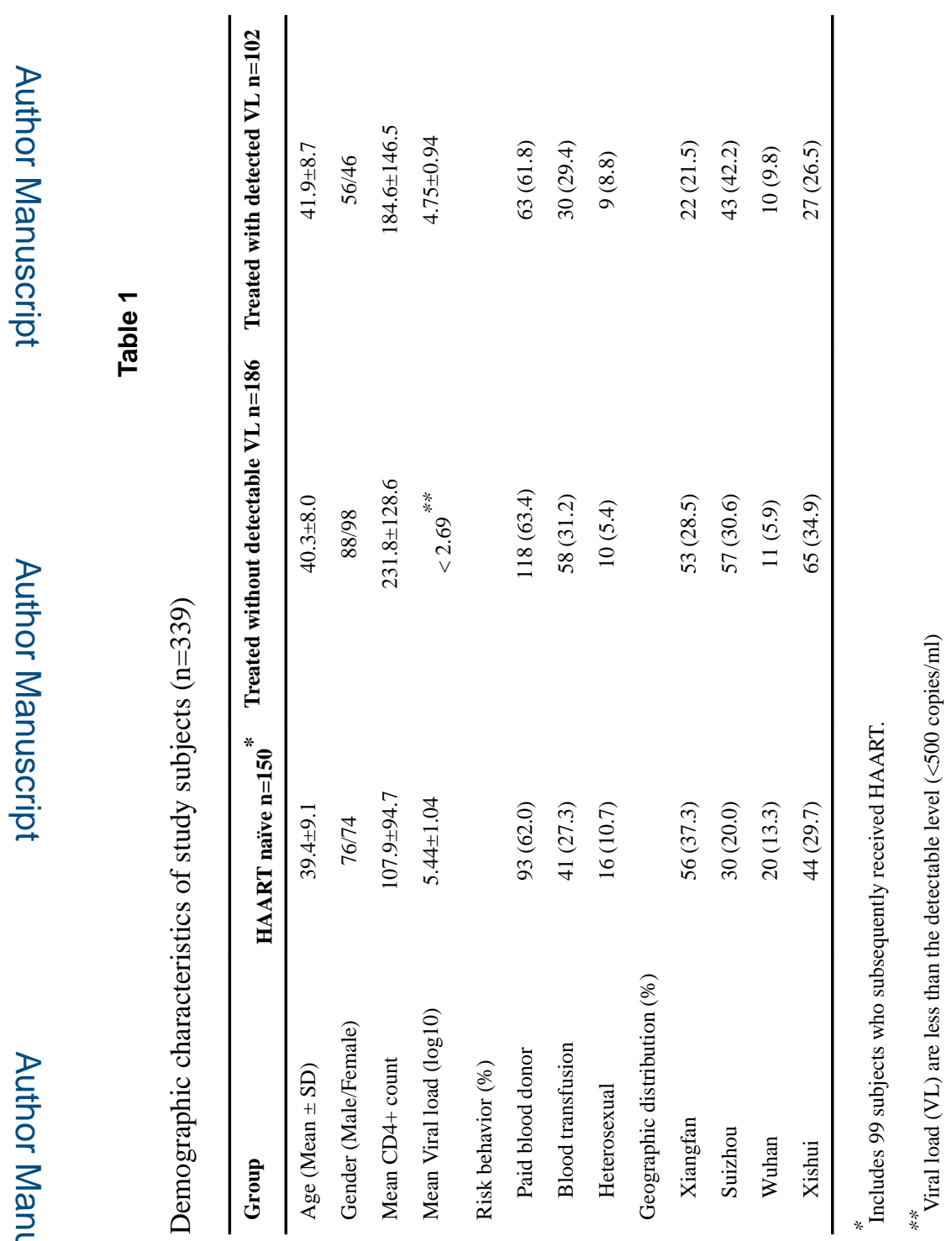



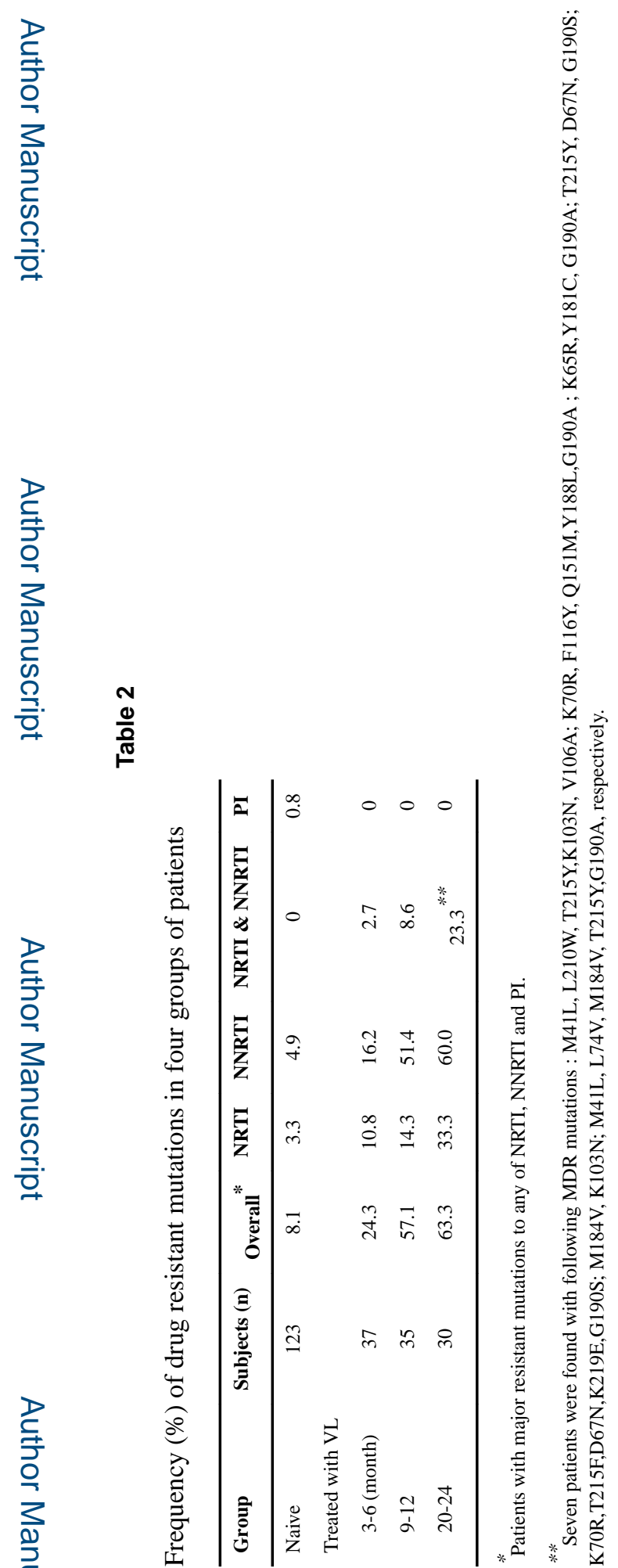

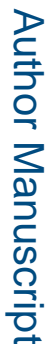

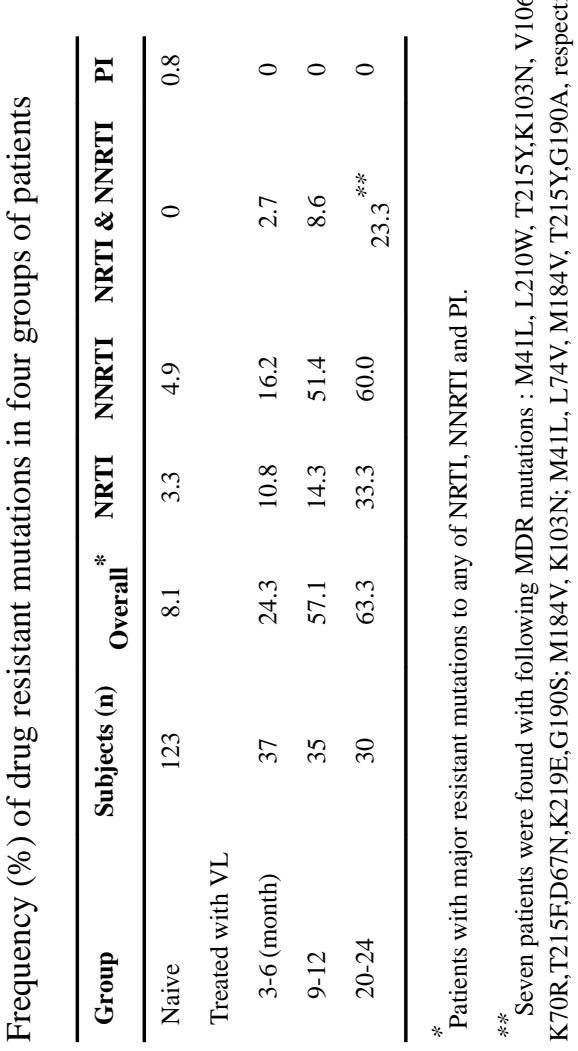


